\title{
Design and Analysis of Auxiliary Calibration Device Based on 3-RPS Parallel Mechanism for Spraying Robot
}

\author{
Youcai ZHANG ${ }^{1, a}$, Jianxiao WANG ${ }^{1, b}$ and Lei QIN $^{2, c}$ \\ ${ }^{1}$ School of Mechatronics Engineering, Foshan University, Foshan, China \\ ${ }^{2}$ Foshan Kingpeng Robotics Technology Co., Ltd, Foshan, China \\ a786516460@qq.com, bjwang90@163.com, ${ }^{\mathrm{c}} 78847713 @ q q . c o m$
}

\begin{abstract}
Keywords: Teaching manipulator, Spraying robot, Parallel mechanism, Kinematic capability, Workspace.

Abstract: According to the calibrating requirements of a teaching manipulator in a glaze-spraying robot system, an auxiliary calibration device based on 3-RPS parallel mechanism was presented. Firstly, the 3-RPS parallel mechanism and other related components were designed. Then the kinematic capability of the device was obtained by forward kinematics. Moreover, the workspace of the device was studied by inverse kinematics and MATLAB software. The results show that the device can meet the required kinematic capability and workspace when calibrating.
\end{abstract}

\section{Introduction}

The process of spraying complex parts, robot needed to be produced planning trajectory movement that would be taught by technician. The teaching manipulator [1] was manually dragged to teach the robot in order to obtain its desired movement. Before teaching, the teaching manipulator must be calibrated, especially the calibration of its parameters. In order to identify parameters conveniently and precisely, parallel mechanism was considered. In recent years, no-full DOF parallel mechanism had attracted many scholars at home and abroad because of its higher accuracy, higher stiffness, compact structure, good bearing capacity. This paper took advantage of its superiority and designed an auxiliary calibration device based on 3-RPS parallel mechanism for spraying robot.

According to the research of 3-RPS parallel mechanism, Hunt [2] proposed 3-RPS parallel mechanism which can move along $\mathrm{Z}$ axis and rotate around $\mathrm{X}, \mathrm{Y}$ axis. In further study, Zhang et al. [3] got the forward kinematics solution of 3-RPS parallel mechanism based on improved particle swarm optimization, Xie et al. [4] used continuous ant colony algorithm to solve the forward kinematics of 3-RPS parallel mechanism, Gao et al. [5] proposed a sphere-coordinate searching method which can be used to analyze the workspace of 3-RPS parallel mechanism, Zhang et al. [6] obtained the workspace of 3-RPS parallel mechanism based on its inverse kinematics and analyzed its deflection capacity. In practical applications, Yan et al. [7] used 3-RPS parallel mechanism to make application for the engine support frame, Song [8] also used 3-RPS parallel mechanism to apply on the oscillating mechanism of welding torch. Cheng et al. [9] designed a support structure of electro-optical tracker system based on 3-RPS parallel mechanism.

In this paper, according to the calibrating requirements of a teaching manipulator in a glaze-spraying robot system, an auxiliary calibration device based on 3-RPS parallel mechanism was presented and the forward kinematics, inverse kinematics of the mechanism were solved. Meanwhile the kinematic capability and workspace of the mechanism were analyzed, which provided the basis of teaching operation for teaching manipulator.

\section{Model of Calibration}

\section{Design of 3-RPS Parallel Mechanism}

At present, the laser measuring instrument was used to calibrate to improve the teaching efficiency and accuracy, such as laser interferometer, laser tracker. However, it had the shortcoming of expensive and high technical threshold. According to the structure of turntable and robot, the complementary 
relationship between serial mechanism and parallel mechanism, this paper presented a 3-RPS parallel mechanism for teaching manipulator. The structure of 3-RPS parallel mechanism as shown in Fig. 1, which was composed of support component, static stage, bearing with box, linear bearing with handle, calibration bar, moving stage, spherical hinge. The mechanism had three chains with identical geometrical condition which can move along $\mathrm{Z}$ axis and rotate around $\mathrm{X}, \mathrm{Y}$ axis by driving robot to control the expansion of its three chains.

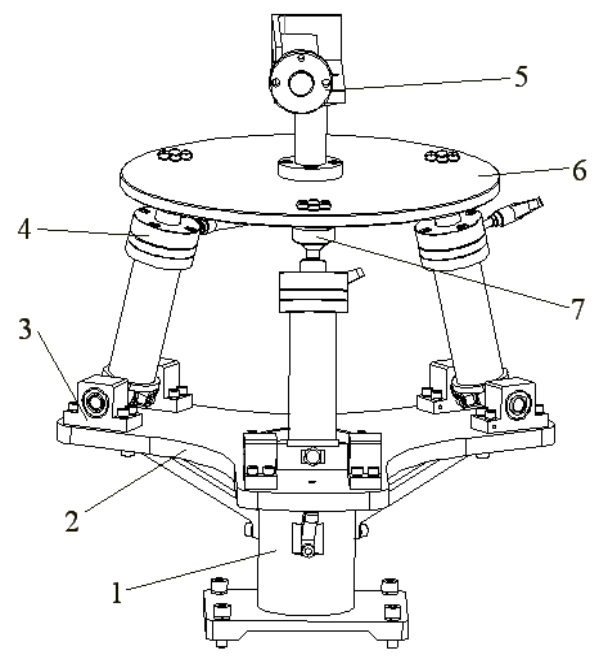

1.support component 2.static stage 3.bearing with box 4.linear bearing with handle 5.calibration bar 6.moving stage 7.spherical hinge

Fig. 1 Structure of 3-RPS parallel mechanism

\section{Calibrating Method}

As described, the calibration integral structure of teaching manipulator as shown in Fig. 2, which was composed of 3-RPS parallel mechanism, turntable, robot, teaching robot manipulator, sliding table. The process of calibration as followed, by conducting the relative calibration of teaching manipulator based on the calibration of robot. 3-RPS parallel mechanism was installed on the turntable, then the robot end-effector was driven to a zone near the moving stage, the moving stage was dragged to match the calibration bar installed on the robot end-effector. Within the range of allowable kinematic capability and workspace, the robot end-effector was driven to a desired pose, The linear bearing with handle was used to immobilize the mechanism, then the angles of robot joints were recorded; the pose of mechanism was fixed and the sliding table was dragged to make teaching manipulator match the calibration bar, the angles of teaching robot manipulator joints were also recorded. The operating process was repeated and the angles under the different poses were gotten which were used to program algorithm to calibrate the parameter of teaching manipulator.

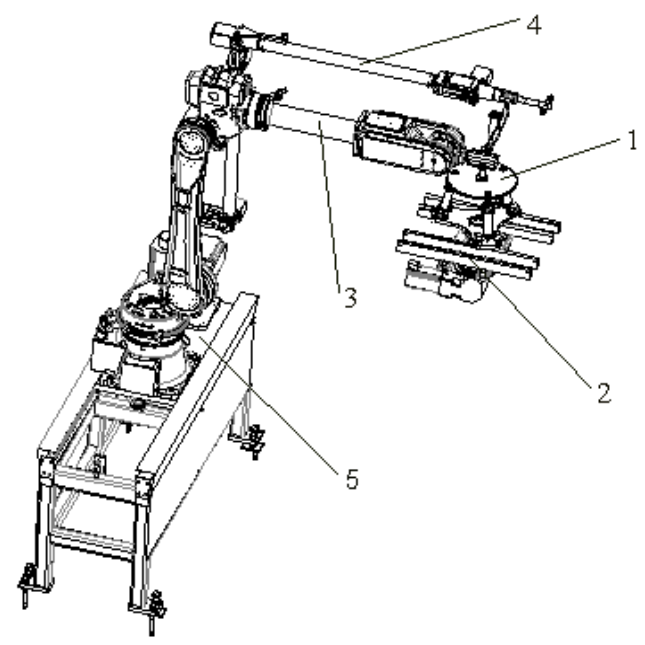

1. 3-RPS parallel mechanism 2.turntable 3.robot 4.teaching manipulator 5.sliding table Fig. 2 Model of calibrating mechanism 


\section{Analysis of Kinematic Capability}

To analyze the 3-RPS parallel mechanism, its kinematic model was proposed by Fig. 3 which included static stage $A_{1} A_{2} A_{3}$, moving stage $B_{1} B_{2} B_{3}$ and three identical RPS kinematic chains. The radius of circumcircle for moving stage and static stage was $r, \mathrm{R}$ respectively.

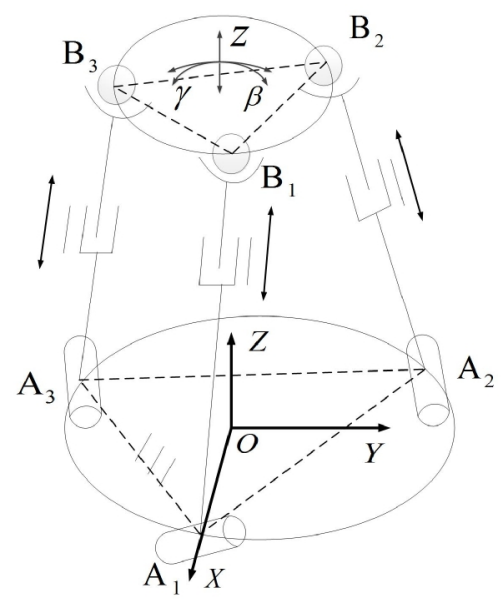

Fig. 3 Kinematic model of 3-RPS parallel mechanism

The fixed coordinate system $O-X Y Z$ was established on the center of static stage and the $X$ axis was pointed to $A_{1}$, the $Z$ axis was perpendicular to static stage. The angles between axis of revolute joint and the $X$ axis were $\theta_{i}=\frac{\pi}{6}(4 i-1),(i=1,2,3)$, the inclined angles between kinematic chains and static stage were $\varphi_{i},(i=1,2,3)$, the position of spherical hinges on the moving stage as shown in Eq. 1.

$$
x_{B_{i}}=X_{A_{i}}+L_{i} \cos \varphi_{i} \cos \left(\theta_{i}-90^{\circ}\right), y_{B_{i}}=Y_{A_{i}}-L_{i} \cos \varphi_{i} \sin \left(\theta_{i}-90^{\circ}\right), z_{B_{i}}=L_{i} \sin \varphi_{i}(i=1,2,3)
$$

The length of equilateral triangle in moving stage $d_{i}$ can be expressed by the points $B_{i}$ and the constraint equation was gotten: $\left|B_{1} B_{2}\right|=\left|B_{1} B_{3}\right|=\left|B_{2} B_{3}\right|=d_{i}$, thus three transcendental equations expressed by $\varphi_{i}$ were gotten. If $x_{i}=\tan \frac{\varphi_{i}}{2}$, then $\sin \varphi_{i}=\frac{2 x_{i}}{1+x_{i}{ }^{2}}$ and $\cos \varphi_{i}=\frac{1-x_{i}{ }^{2}}{1+x_{i}{ }^{2}}$, the angles $\varphi_{i}$ can be solved. The moving coordinate system origin was derived as followed Eq. 2 .

$$
x_{c}=\frac{1}{3} \sum_{i=1}^{3} x_{B_{i}}, y_{c}=\frac{1}{3} \sum_{i=1}^{3} y_{B_{i}}, z_{c}=\frac{1}{3} \sum_{i=1}^{3} z_{B_{i}}
$$

So that the direction cosine of three coordinate axes in moving coordinate system was given by Eq. 3 .

$$
x=\frac{1}{\left|c B_{1}\right|}\left[\left[\left(x_{B_{1}}-x_{c}\right)\right] i+\left[\left(y_{B_{1}}-y_{c}\right)\right] j+\left[\left(z_{B_{1}}-z_{c}\right)\right] k\right], y=\frac{1}{\left|B_{3} B_{2}\right|}\left[\left[\left(x_{B_{2}}-x_{B_{3}}\right)\right] i+\left[\left(y_{B_{2}}-y_{B_{3}}\right)\right] j+\left[\left(z_{B_{2}}-z_{B_{3}}\right)\right] k\right], z=x \times y
$$

In the Eq.3, $\left|c B_{1}\right|=\sqrt{\left(x_{B_{1}}-x_{c}\right)^{2}+\left(y_{B_{1}}-y_{c}\right)^{2}+\left(z_{B_{1}}-z_{c}\right)^{2}},\left|B_{3} B_{2}\right|=\sqrt{\left(x_{B_{2}}-x_{B_{3}}\right)^{2}+\left(y_{B_{2}}-y_{B_{3}}\right)^{2}+\left(z_{B_{2}}-z_{B_{3}}\right)^{2}}$.

In this paper, the parameters of 3-RPS parallel mechanism as followed, the radius of circumcircle for moving stage $r=122 \mathrm{~mm}$ and static stage $R=162 \mathrm{~mm}$, the range of extensible link from $10 \mathrm{~mm}$ to $90 \mathrm{~mm}$. In the initial state, the vertical distance between moving stage and static stage was $210 \mathrm{~mm}$. In order to analyze the kinematic capability of 3-RPS parallel mechanism, three types of limiting pose as shown in Fig. 4. 


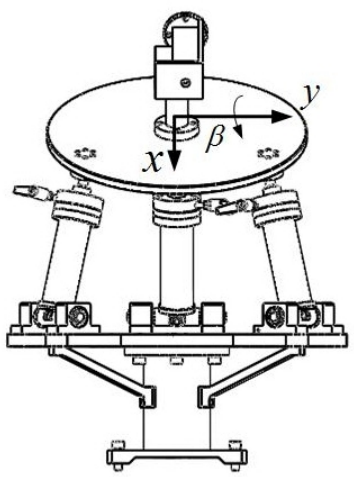

$\mathrm{L}=\left[\begin{array}{lll}90 & 10 & 10\end{array}\right] / \mathrm{mm}$

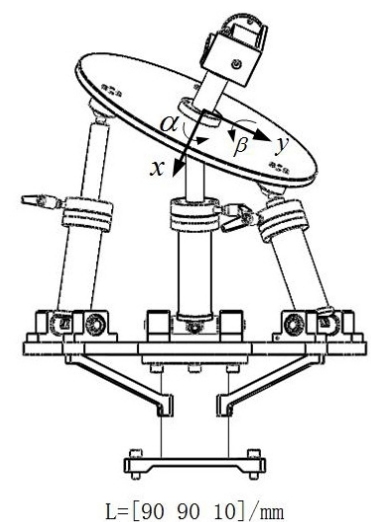

$\mathrm{L}=\left[\begin{array}{lll}90 & 90 & 10\end{array}\right] / \mathrm{mm}$

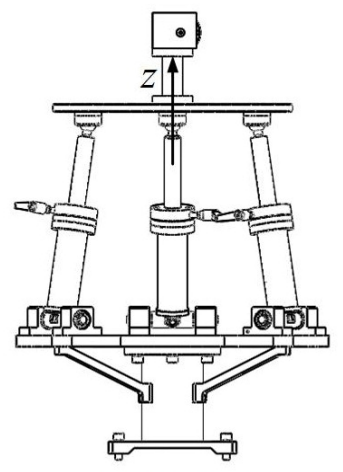

$\mathrm{L}=\left[\begin{array}{lll}90 & 90 & 90\end{array}\right] / \mathrm{mm}$

Fig. 4 Limiting pose under different range

According to the limiting pose of 3-RPS parallel mechanism, its kinematic capability can be shown in Table 1. From the Table 1, 3-RPS parallel mechanism showed that it had kinematic capability of moving along $z$ axis from $210 \mathrm{~mm}$ to $291.5 \mathrm{~mm}$, rotating around $x$ axis 16.4 degree and $y$ axis 18.9 degree, which satisfied the kinematic capability when calibrating.

Table 1 kinematic capability of 3-RPS parallel mechanism

\begin{tabular}{cc}
\hline pose & $(\gamma, \beta, z)$ \\
\hline range of link & $0^{\circ}, 18.9^{\circ}, 246.3 \mathrm{~mm}$ \\
{$[90,10,10] /[\mathrm{mm}]$} & $16.4^{\circ}, 9.5^{\circ}, 272.9 \mathrm{~mm}$ \\
{$[90,90,10] /[\mathrm{mm}]$} & $0^{\circ}, 0^{\circ}, 291.5 \mathrm{~mm}$ \\
\hline $90,90,90] /[\mathrm{mm}]$ & \\
\hline
\end{tabular}

\section{Analysis of workspace}

The workspace of parallel mechanism is the spatial regions of the moving stage origin can be reached. In this paper, the workspace of 3-RPS parallel mechanism was the operating area of the robot end-effector, which based on the inverse kinematics considering constraints about length of link, angle of kinematic pair, interference of link, and the analysis was used MATLAB software to simulate the workspace. The pose of moving stage was adopted $X-Y-Z$ fixed angle to indicate $(\alpha, \beta, \gamma)$, which transformation matrix as shown in Eq. 4.

$$
T=\left[\begin{array}{cccc}
c \beta c \gamma & s \beta c \gamma s \alpha-s \gamma c \alpha & s \beta c \gamma c \alpha+s \gamma s \alpha & x_{c} \\
s \gamma c \beta & s \gamma s \beta s \alpha+c \gamma c \alpha & s \gamma s \beta c \alpha-c \gamma s \alpha & y_{c} \\
-s \beta & c \beta s \alpha & c \beta c \alpha & z_{c} \\
0 & 0 & 0 & 1
\end{array}\right] \Rightarrow T=\left[\begin{array}{cccc}
x_{i} & y_{i} & z_{i} & x_{c} \\
x_{j} & y_{j} & z_{j} & y_{c} \\
x_{k} & y_{k} & z_{k} & z_{c} \\
0 & 0 & 0 & 1
\end{array}\right]
$$

In the Eq. 4, s $\alpha=\sin \alpha, s \beta=\sin \beta, s \gamma=\sin \gamma c \alpha=\cos \alpha, c \beta=\cos \beta, c \gamma=\cos \gamma$.The positions of $B_{i}$ on the moving coordinate system were expressed by Eq. 5 .

$$
{ }^{c} B=\left[\begin{array}{ccc}
r & -\frac{r}{2} & -\frac{r}{2} \\
0 & \frac{\sqrt{3} r}{2} & -\frac{\sqrt{3} r}{2} \\
0 & 0 & 0 \\
1 & 1 & 1
\end{array}\right]
$$

Then the positions of $B_{i}$ on the fixed coordinate system can be derived by ${ }^{o} B=T \square^{c} B$. According to the constraint planes: $y=0, y=-\sqrt{3} x, y=\sqrt{3} x$, three constraint formulas can be gotten: 
$y_{c}=-x_{j} r, x_{j}=y_{i}, x_{c}=\frac{r}{2}\left(x_{i}-x_{j}\right)$. So that $x_{c}, y_{c}, \gamma$ can be solved as shown Eq. 6 , the transformation matrix would be included only three parameters.

$$
\left\{\begin{array}{c}
\gamma=\arctan \frac{\sin \alpha \sin \beta}{\cos \alpha+\cos \beta} \\
x_{c}=\frac{r}{2}(\cos \beta \cos \gamma-\sin \alpha \sin \beta \sin \gamma) \\
y_{c}=r(\cos \alpha \sin \gamma-\cos \gamma \sin \alpha \sin \beta)
\end{array}\right.
$$

So the length of extensible link can be expressed as $l=\left|{ }^{o} B-{ }^{o} A\right|,{ }^{o} A$ was the coordinate matrix of the points $A_{i}$ on the fixed coordinate system.

$$
{ }^{o} A=\left[\begin{array}{ccc}
R & -\frac{R}{2} & -\frac{R}{2} \\
0 & \frac{\sqrt{3} R}{2} & -\frac{\sqrt{3} R}{2} \\
0 & 0 & 0 \\
1 & 1 & 1
\end{array}\right]
$$

In order to analyze the workspace of 3-RPS parallel mechanism, using MATLAB software to program numerical searching to solve discrete points in the workspace and plot the fitting workspace. The process of fitting as follows: from the moving stage initial position to get the section parallel to the plane $X O Y$, the range of section was $z_{c \min } \leq z_{c} \leq z_{c \max }$; the inclined angle $\gamma$ and $\beta$ can be expressed as followed: $\gamma=\rho \cos \theta, \beta=\rho \sin \theta, \rho \in[0, \pi], \theta \in[0,2 \pi]$. To set the given step for $z_{c}, \theta, \rho$ and solve the inverse kinematics; add the constraints about length of link: $l_{\min }<l<l_{\max }$ and angles of kinematic pair: $\varphi_{\min }<\varphi<\varphi_{\max }$, determine whether each group $\gamma, \beta$ was meaningful; change the section $z_{c}$, search all of section from $z_{c \min }$ to $z_{c \max }$ until whole workspace was searched and get the values set of $\gamma, \beta$.

In this paper, the parameters of 3-RPS parallel mechanism as followed, the radius of circumcircle for moving stage $r=122 \mathrm{~mm}$ and static stage $R=162 \mathrm{~mm}$, the maximum length of extensible link $l_{\max }=294 \mathrm{~mm}$, the minimum length of extensible link $l_{\min }=214 \mathrm{~mm}$, the allowable angle of revolute joint and spherical joint was respectively 80 degree, 32 degree. According to the numerical searching method, the workspace of 3-RPS parallel mechanism as shown in Fig. 5.

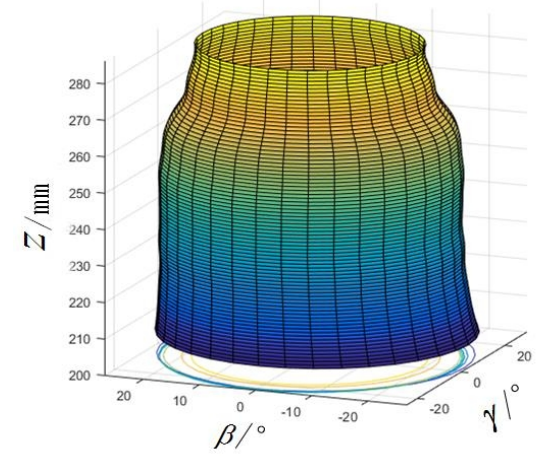

Fig. 5 Workspace of 3-RPS parallel mechanism 
The workspace of mechanism was varied with the section height and had symmetric structural properties. The workspace of teaching manipulator was bigger than the workspace of 3-RPS parallel mechanism, so to avoid the operating area on the process of calibrating, making sure the workspace had practical significance for robot end-effector.

\section{Conclusions}

(1)This paper was proposed an auxiliary calibration device based on 3-RPS parallel mechanism which had the character of compact structure and light. At the meantime, with the advantages of serial and parallel structure, the manipulator can follow the robot movement and meet the needs of teaching calibration.

(2)The forward kinematics was solved, which showed that the mechanism can achieve moving along $z$ axis $81.5 \mathrm{~mm}$, rotating around $x$ axis 16.4 degree and $y$ axis 18.9 degree, which satisfied the kinematic capability when calibrating.

(3)The inverse kinematics was solved, the workspace of mechanism was presented and the operating area of teaching robot manipulator was determined.

\section{Acknowledgements}

This work was financially supported by the Technological Innovation Program of Foshan City, Guangdong, China.

\section{References}

[1] Yu XY, Qin L, Zhou WP. An Automatic Spraying Articular Arm without Power. CN103351177A [P] (2013). (In Chinese)

[2] Hunt K H. Structural Kinematics of In-Parallel-Actuated Robot-Arms [J]. Journal of Mechanical Design, 105(4):705-712 (1983).

[3] Zhang W, Gao H, Chen Y et al. Forward Kinematics Study of 3-RPS Parallel Mechanism Based on Improved Particle Swarm Optimization [J]. Journal of Jinggangshan University, 37(4):63-67 (2016). (In Chinese)

[4] Xie ZJ, Liang H, Song DP. Forward Kinematics of 3-RPS Parallel Mechanism Based on a Continuous Ant Colony Algorithm [J]. China Mechanical Engineering, 26(6):799-803 (2015). (In Chinese)

[5] Gao F, Huang YM. The Sphere-Coordinate Searching Method of the Workspace Analysis of the 3-RPS Parallel Mechanism [J]. Journal of Xi an University of Technology, 17(3):239-242 (2001). (In Chinese)

[6] Zhang LJ, Lei C, Guo M et al. Deflection Capacity Analysis of Moving Platform of a 3-RPS Parallel Mechanism [J]. Journal of Yanshan University, 36(3):196-200 (2012). (In Chinese)

[7] Yan S, Li Y, Jiang XB et al. The Design of Motor Bench Scale Based on 3-RPS Parallel Mechanisms [J]. Journal of Shandong Traffic University, 17(1):71-73 (2009). (In Chinese)

[8] Song X. Analysis on Application of Welding Gun Oscillation Mechanism to 3-RPS Parallel Mechanism [J]. Transactions of The China Welding Institution, 2004(4):24-26 (2004). (In Chinese)

[9] Chen G, Wang YJ, Ge SR. Error Sensitivity of 3-RPS Symmetrical Parallel Supporting Structure [J]. Opto-Elec Eng, 36(9):146-150 (2009). (In Chinese) 